\title{
Development of a Kinematic Human Model for Clothing and High Performance Garments
}

\author{
Doudou ZHANG, Sybille KRZYWINSKI \\ TU Dresden, Institute of Textile Machinery and High Performance Material Technology, Germany
}

https://doi.org/10.15221/19.068

\begin{abstract}
In recent years, there have been significant efforts directed towards the 3D product development of clothing on virtual human models. However, developers legitimately criticize that static human models are not suitable for the construction of sports, medical, and protective clothing. A garment that fits a static shape may be very uncomfortable while performing daily tasks including walking, sitting, or reaching. Without a realistic body shape and natural human postures, it is difficult to create properly fitting apparel. To improve wearing comfort and shorten development times, clothing must be designed based on specific body postures. Moreover, innovative knowledge about the interaction between body and garment during movement must be considerate.
\end{abstract}

Keywords: animation, 3d scan, fitting simulation, human model

\section{Introduction}

Presently available solutions for the assessment of wearing comfort during movement are based on virtual human models. However, currently, there is no human kinematic model for the display of realistic body deformations during movement, especially in the areas of elbow, knee, and hip joint. The resulting unrealistically deformed and partially penetrating surfaces do not allow for the creation of tight fitting garments in 3d. Especially when wearing functional and sports clothing, typical body positions strongly deviate from standard scanning postures. Hence, the generation of high-quality kinematic human models is of great importance in this research context.

In order to overcome the issues addressed above, an efficient and direct procedure for the development of realistic, personalized, kinematic virtual human models must be created, using only one $3 \mathrm{~d}$ scan properly reflecting the anatomy of the human body. To achieve this aim, a template for skin, skeleton, and muscles is provided. A methodology for the application of the kinematic model onto individual scan data is developed, movement and specific body postures of human models are generated, and clothing test samples are designed as well as simulated on animated scans through the software Clo3d. Garment deformation is no longer investigated exclusively based on a static body shape, but also based on a wide variety of natural human poses and dynamic body deformations.

\section{Methods}

In this research, we investigated the generation of individual, high-quality, kinematic human models, which can be integrated into digital process chains for the development of functional clothing. We present different techniques for automatically generating a personalized kinematic human model regarding speed, fidelity, compatibility, and software availability. Specifically, the following methods are compared:

- Linear blend skinning in simulation software Clo3d [1],

- Auto-rigging of $3 \mathrm{~d}$ scan on online service Maximo [2],

- Skinned Multi-Person Linear (SMPL) [3] human model based on data and

- Anatomical simulation with plugin Ziva dynamics [4].

\subsection{Linear blend skinning}

The main advantage of Linear Blend Skinning (LBS) is the fact that it is not computationally intensive, yet still provides acceptable deformations in a good range of situations. Basic linear blend skinning models are the most widely used and are supported by most $3 \mathrm{~d}$ simulation software solutions. In order to convert a body scan into an avatar adapted the body shape, the function named "convert to avatar" in the 3d simulation software Clo3d was employed (see figure 2). Avatar joints are organically linked allowing for easier modification of poses. For the simulation, the basic construction of a pair of men's trousers and a T-shirt were selected (see figure 2), and simulations were performed taking into account the mechanical material behavior. The strain map (see figure $2 \mathrm{~b}, \mathrm{c}$ ) is a geometrical measurement of 
the garment distortion. This presentation demonstrates how the pattern cuts deform while being worn. Thus, this serves as a good indicator as to whether fabric needs to be added to or removed from certain parts of the garment.

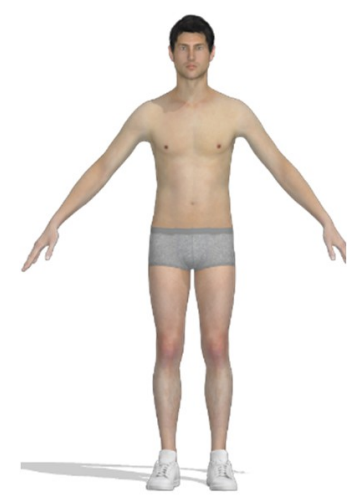

a

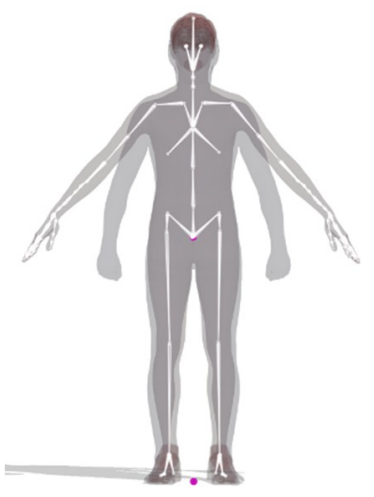

$\mathrm{b}$

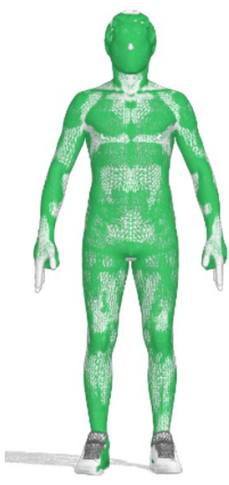

C

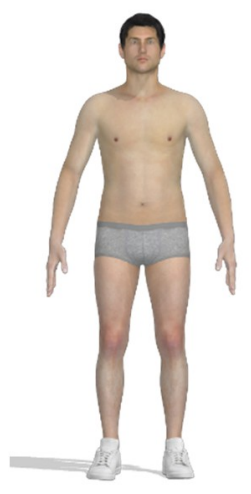

d

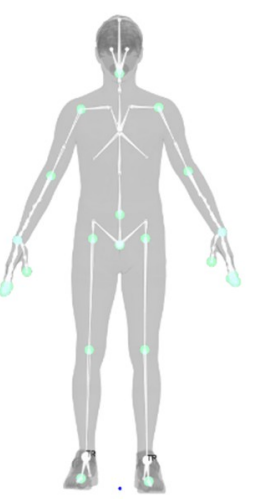

e

Fig. 1. Workflow of conversion to avatar: a. Clo avatar; b. align $3 d$ body scan and Clo avatar; c. align avatar joints; $d$. converted avatar; e. $3 d$ body scan with joints.

The same texture mapping blurs the geometric differences between the avatar and the scan (see figure $1 a-$ Clo3d avatar, 1d - converted avatar).

However, there are also disadvantages to LBS. The body shapes are "approximated" by the adaptation of the scanning mesh to a standard mesh with significantly lower resolution. This mainly affects the volume loss in the joints, called the "candy wrapper effect", when bending and twisting. Unrealistic deformation in the areas of elbow, knee, and hip joint (see figure $2 \mathrm{~d}$ ) cannot be overcome with this method. So far, the fit assessment was primarily done in the standing position and while walking.

As a result, fitting simulations for loose fitting garments on a converted Clo3d avatar are sufficient since extreme postures are neglected.

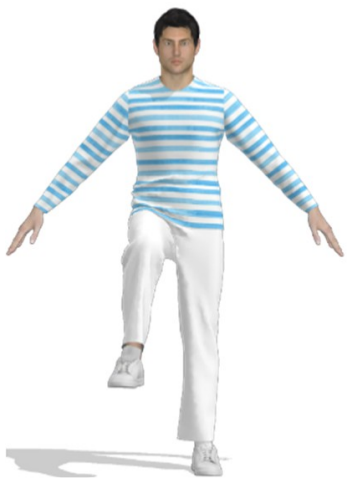

a

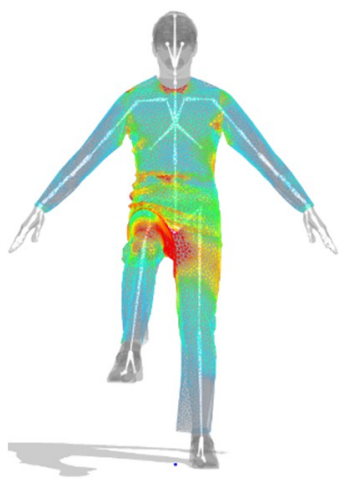

b

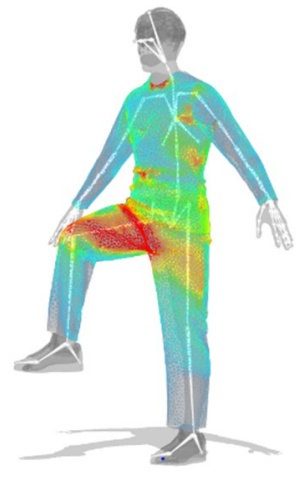

$\mathrm{C}$

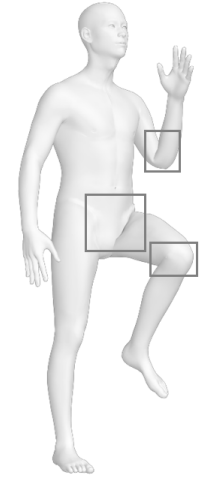

d

Fig. 2. Fitting simulation: a. individual avatar with bent leg; b,c. assessment of fit with strain map ( frontal and right view); d. unrealistic deformation in the areas of elbow, knee, and hip joint

\subsection{Rigging of a $3 d$ scan}

In this section we would like to introduce a more accurate animation method that based on the use of the original scan data and requires very little $3 d \mathrm{CAD}$ or animation knowledge.

To show movements based on 3d scan data, we used the Mixamo auto-rigging online service, which calculates the skinning weights and inserts animation structures (joints, bones) into the $3 \mathrm{~d}$ scan (see figure 3). For this purpose, it is necessary to align the scan data exactly in the frontal plane and to mark the position of the joints interactively.

From a database, recorded movements (motion capture data) can be selected (walking, running).

These movements are transferred to the skeleton inserted into the scan data. This leads to a more realistic deformation of the body surface during movement. Figure $3 f$ shows the improved deformation of the skin surface around the joints. Overlaps of the surface are not completely avoided (hip joint).To test the interaction between the animated model and the garment a fit simulation program, e.g. Clo3d, used. The model has to be imported in FBX format. 


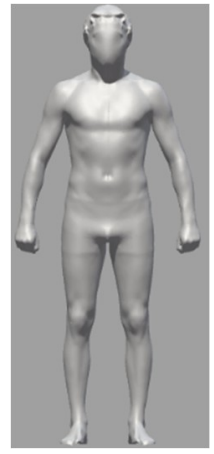

a

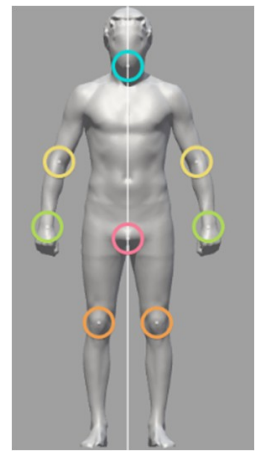

$\mathrm{b}$

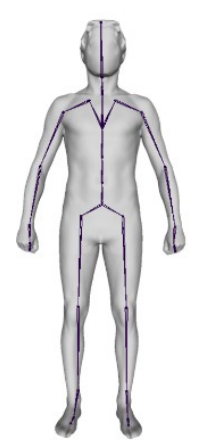

C

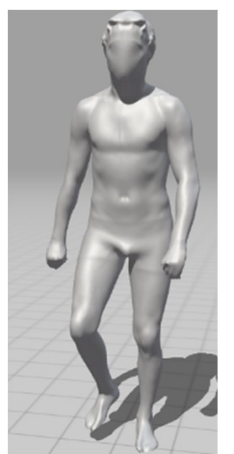

d

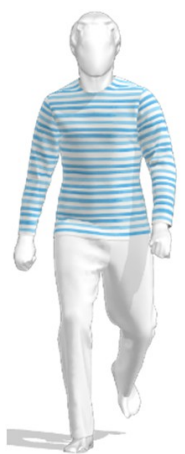

e

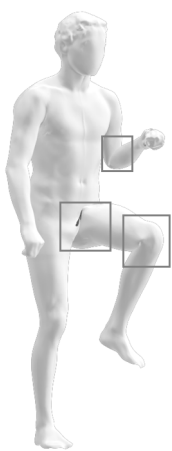

f

Fig. 3. Auto-rigging: a. 3d scan surface; b. place makers; c. insert skeleton structure;

d. generation of animated $3 d$ avatar; e. interaction between garment and body during movement;

f. improved deformation in the areas of elbow, knee, and hip joint

\subsection{Data-driven simulation}

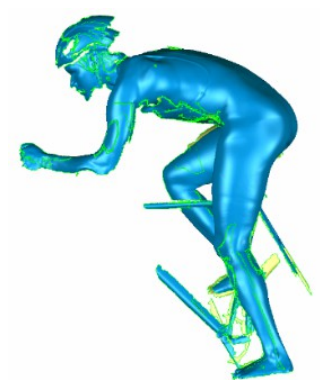

Fig. 4. 3d scan with MHT scanner form Artec [5]

In this part of the research, a cycling posture was derived from san data in a T position (see figure 5a), passing through different positioning angles and ends with a specified cycling position. In order to obtain an "kinematic body model", scan data of a professional cyclist (see figure 4) were combined with a "kinematic template model" in Maya program (see figure a-c). Maya [6] is a 3d modeling and rendering software that helps to create virtual scenes for design visualizations and computer games. Such models are needed, to consider the flow behavior around the cyclist in wind tunnel test or for simulation-based analyses.

For this study a kinematic body model (SMPL) [3] developed by the Max Planck Institute was used. This is rated excellent in the literature. It makes it possible to represent a large number of body poses realistically and to transfer them to scan data. The calculation of the occurring deformations of the body is based on a huge database and trained from thousands of $3 \mathrm{~d}$ scans. In addition, the research presented here made it possible to merge them with the scan data of a cyclist (see figure 4) by adapting the template surface to the surface of the target scan model.

For the adaption of $3 \mathrm{~d}$ scan data into the SMPL model, two conditions must be met: T-position (see figure $5 \mathrm{a}$ ) and the same topology as SMPL (see figure $5 \mathrm{~b}$ ). To merge SMPL model and $3 \mathrm{~d}$ scan, both models must be imported into Maya.
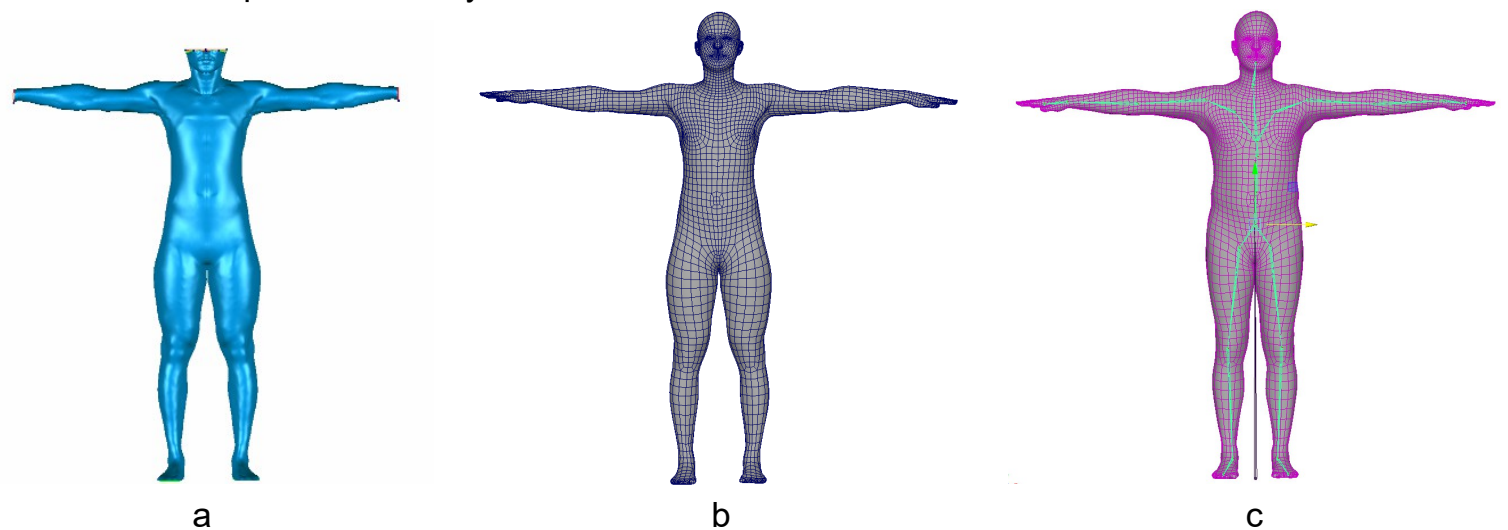

Fig. 5. a: 3d scan in T position; b: surface mesh with the same topology as SMPL model based on 3d scan; c: SMPL model (green: skeleton; pink: body surface) 
The next step is the application of the Maya tool BlendShape. BlendShape is a technique to deform a mesh in to predefined shapes. In our study, the $3 d$ scan of the cyclist is the predefined shape. The SMPL template can changed based on the $3 d$ scan. If you add the target mesh (see figure $5 b$ ) to m_avg_blendshapes in the Add Blend Shape Target Options window, the scanned model is integrated as a shape into the SMPL model, that can be viewed at Shape Editor (see figure 6a) and the shape is displayed as a numerical value in the range 0.000 to 1.000 , where 0 is the basic shape of the SMPL model and 1 is the shape of our scanned model. This converts the SMPL model into a new model that represents the $3 \mathrm{~d}$ scan body shape and the mesh structure of the SMPL model, allowing us to create a variety of poses with this converted model (see figure 6). But the skeleton of the converted model is still not accurate because it shows the old skeleton of the SMPL base model. To solve the skeleton problem of the converted model, the plugin SMPL - Rigging \& Pose Corrections Toolbox for Maya is used, and the skeleton is corrected with the Recompute Skeleton function of this plugin. The converted model, the mesh and the correct skeleton are shown in (see figure 6c).

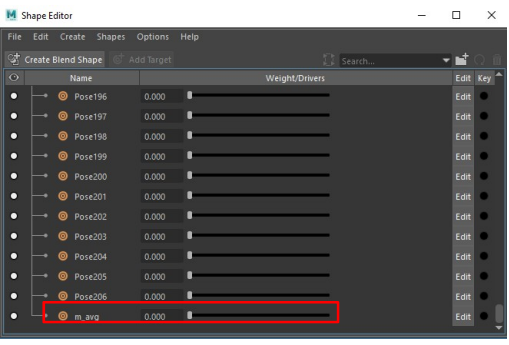

a
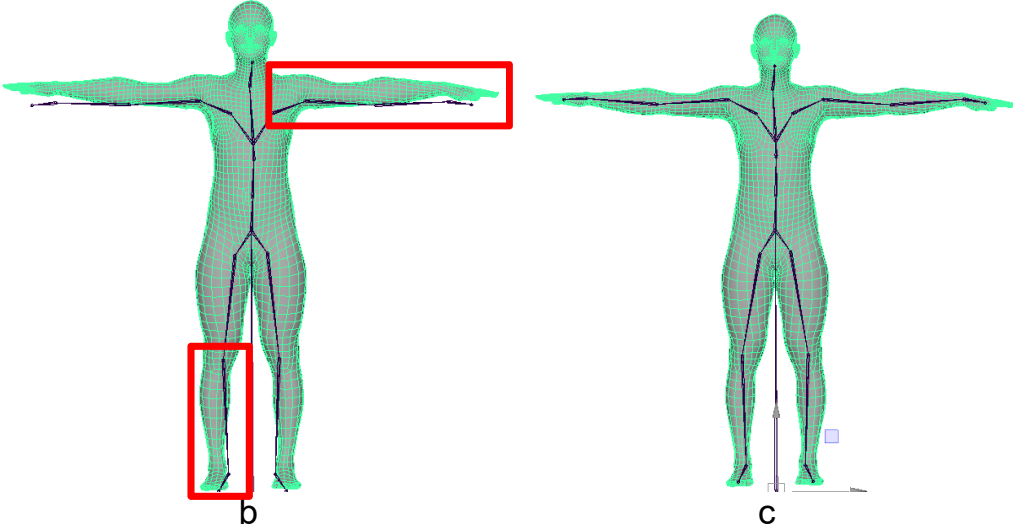

Fig. 6. a: add blend shape target options; b: shape editor; c: converted kinematic human model based on 3d scan; $d$ : optimized skeleton using 'Recompute Skeleton' feature

Now an animatable scan is available to generate the cycling position. With the tools Move and Rotate the joints of the skeleton can be controlled to reach the user defined cycling position of the $3 \mathrm{~d}$ scan (see figure 7). The deformation of the body is done by clicking the function Apply Pose Blend Shapes to Current Frame of the plugin. After this step, the deformation of the body is visually checked for each time to ensure that no unusual deformation problems occur.

It was found that there are differences in dimensions between scanned and kinematic human models (see figure 7). It was obvious that the mesh was not sufficiently and proportionally deformed. One reason that has led to unrealistic deformations is the simple skeletal structure; another factor is soft tissue deformation, which is not applicable to muscle deformations.
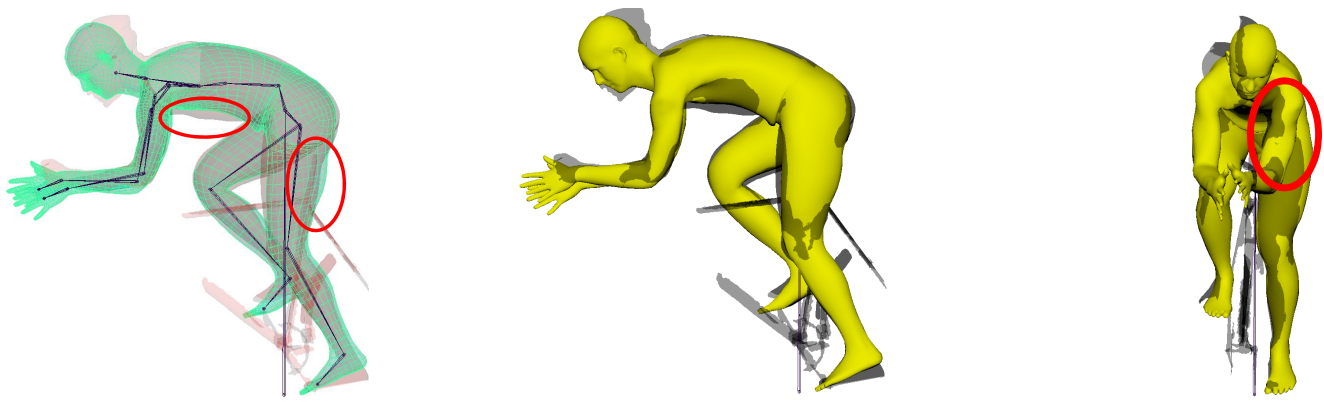

Fig. 7. Comparison between 3d scan (gray) and kinematic body model (yellow).

To improve the result, the wrapping technique was used. The mesh of the kinematic human model in cycling position is considered as base mesh. The processing of the mesh surfaces was done in the software Wrap3 [7]. The process is divided into several steps. Each step generates a set of control nodes in the base mesh with a specific density that increases with each new step. The algorithm attempts to determine the position of the control nodes so that the base mesh comes as close as possible to the target geometry. Once a solution has been found, the next step is taken. It scans the base mesh with a larger number of control nodes and repeats the adjustment. The greater the number of control nodes in the current step, the more accurate the result. 


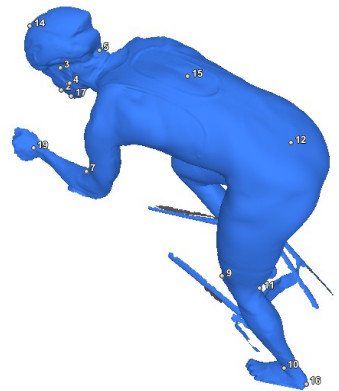

a

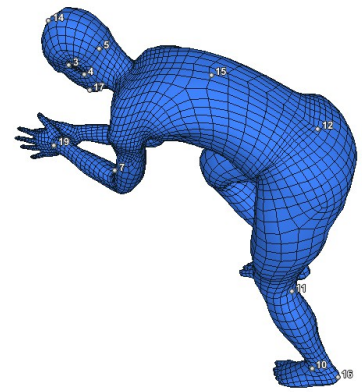

b

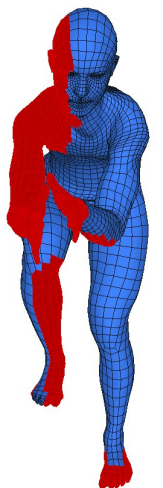

C

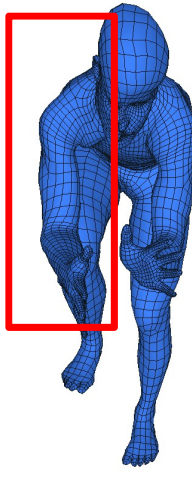

d

Fig. 8. a: 3d scan; b: cycling posture simulation using a kinematic template model; c: selected polygon; $d$ : wrapping result.

After wrapping, there are still some issues in the right side of upper body, which could be rebuilt in the next step (see figure 8d).

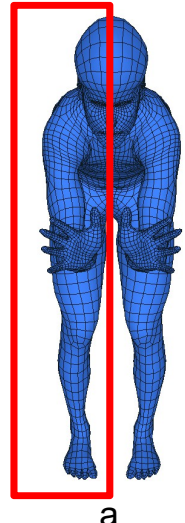

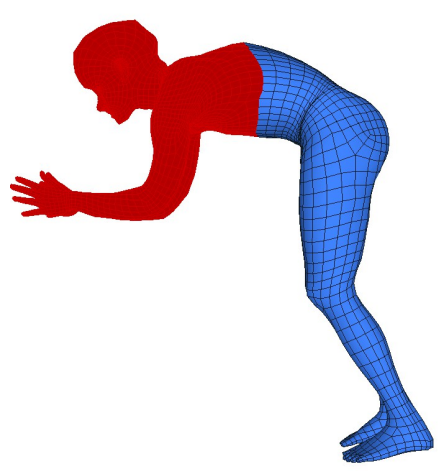

b

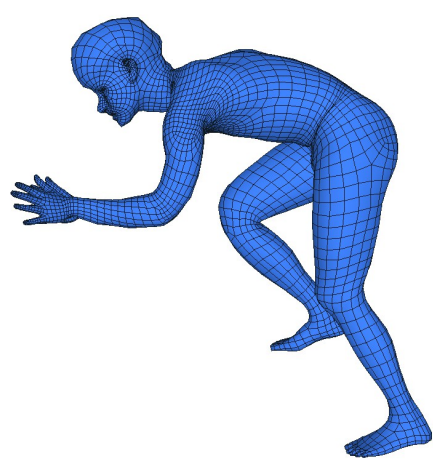

C

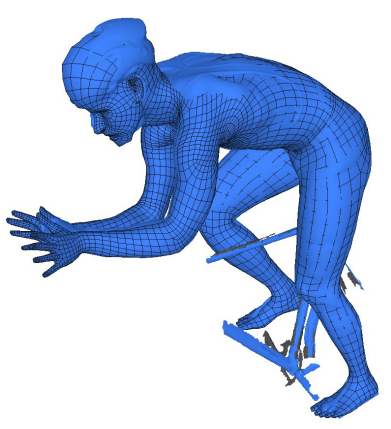

d

Fig. 9. a: fix symmetry; b: selection of a set of polygon (red), called B model; c: replace the lower trunk and the legs; $d$ : alignments between scan data and reconstructed body model.

In this cycle position, the upper body is assumed to be symmetrical, so that the symmetry on the model was determined from the last step. On the other hand, the legs are asymmetric, so the lower body has been replaced by the model, pictured in see figure $8 \mathrm{~d}$. In the last step the two models in the same topology are taken and seamlessly replaced the selected part of the B model with the part of the predecessor model.

\subsection{Anatomical simulation}

For the anatomical simulation we used an anatomical model from the commercially available Ziva dynamics. This $3 \mathrm{~d}$ anatomy model was created with the help of a physically based simulation. It provides an optimized interface to simulate the width of anatomical tissues responsible for the physical appearance of a human body [4]. Compared to the data driving method, physical simulation offers the advantage of building personal bones and muscles (see figure $10 \mathrm{a}-\mathrm{c}$ ), which function differently in animations [8]. Skin deformation is based on the underlying muscle that solves the problem in the elbow, knee and hip joint areas.

Unfortunately, the construction of an anatomically realistic avatar with a personal scan is a labor- and computationally-intensive procedure and very difficult for clothing experts. Here we tested this $3 d$ anatomy model imported into Clo3d and designed protective clothing on it. The muscle deformation also affects the deformation of garments that can be selected as a new design component. A worker in protective clothing should therefore be able to perform the constant movements effortlessly and always be comfortably dressed. 


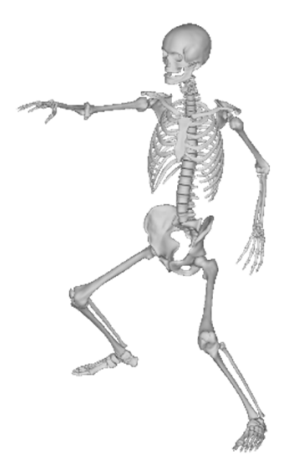

a

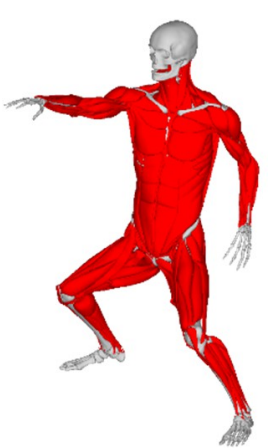

b

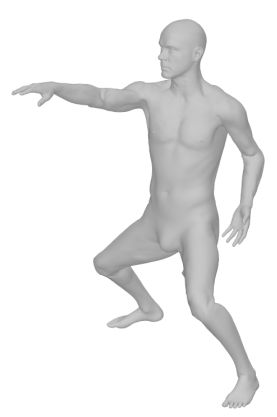

C

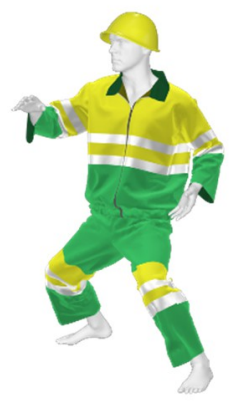

d

Fig. 10. physical simulation: a. bones; b. muscles; c. skin; $d$. simulation of functional clothing.

\section{Conclusion}

For the development of functional clothing, it is essential to be able to visualize these garments on individual human models and to be able to check the fit. For this purpose, kinematic human models are needed, which can be derived from scan data or generated anatomy-based. In this publication, various possibilities of model generation will be tested and evaluated concerning their applicability for close- or loose-fitting garments. Besides, a first assessment is made of the applicability of such models in the context of a virtual process chain for clothing development.

The degree of detail and the procedure for creating the models vary greatly. Thus, it is necessary to define the requirements in advance of product development to select the right development methodology.

\section{References}

[1] "CLO |3D Fashion Design Software," CLO Official Site. [Online]. Available: https://www.clo3d.com. [Accessed: 08-Aug-2019].

[2] "Mixamo." [Online]. Available: https://www.mixamo.com/\#/. [Accessed: 08-Aug-2019].

[3] "MPI SMPL." [Online]. Available: http://smpl.is.tue.mpg.de/. [Accessed: 08-Aug-2019].

[4] "Ziva Dynamics | Home." [Online]. Available: https://zivadynamics.com/. [Accessed: 08-Aug-2019].

[5] "3D-Objektscanner Artec Eva | Der Beste Strukturlichtscanner." [Online]. Available: https://www.artec3d.com/de/portable-3d-scanners/artec-eva. [Accessed: 08-Aug-2019].

[6] "Maya | Software für Computeranimation und Modellierung | Autodesk." [Online]. Available: https://www.autodesk.de/products/maya/overview. [Accessed: 08-Aug-2019].

[7] "R3DS | Russian3DScanner." .

[8] P. Kadleček, A.-E. Ichim, T. Liu, J. Křivánek, and L. Kavan, "Reconstructing Personalized Anatomical Models for Physics-based Body Animation," ACM Trans. Graph., vol. 35, no. 6, pp. 213:1-213:13, Nov. 2016. 